\title{
From bench to bedside and back again?
}

T

The New England Fournal of Medicine recently published the results of a clinical study involving 9 patients with non-small-cell lung cancer who responded to the drug gefitinib. ${ }^{1}$ Nine patients? How important can such a study be? The research arose from a lively area of investigation into the regulatory mechanisms of cell proliferation and growth. Gefitinib is one of a new generation of chemotherapeutic agents aimed at specific molecular targets - in this case, the epidermal growth factor receptor, a genetically modulated receptor that, when stimulated, promotes cell proliferation. ${ }^{2}$

Initial clinical trials of gefitinib in combination with chemotherapy showed no additional benefit of gefitinib for patients with advanced non-small-cell lung cancer. However, the results did show considerable variability: patients in Japan had higher response rates than Europeans, and, among American patients, women, nonsmokers and those with adenocarcinoma were more likely to respond than men. Postulating that these differences could have a genetic basis, a group of scientists compared tumour lines from Japanese and American patients. They found somatic mutations in the tumours that caused differences in the epidermal growth factor receptor - differences that occurred more frequently in Japanese patients, women and those with adenocarcinoma.

Similarly, the authors who reported in the New England Fournal of Medicine took another look at the results in 9 patients with advanced, chemotherapy-refractory nonsmall-cell lung cancer who responded to gefitinib. Finding that 8 possessed the epidermal growth factor receptor mutation, they concluded that "screening for such mutations in lung cancers may identify patients who will have a response to gefitinib."

The concept of studying a small subset of patients is intriguing in this age of large, population-based, multicentre randomized clinical trials (RCTs). Such trials are the touchstone of evidence-based medicine. But here is an observational, nonrandomized study of 9 highly selected patients in which bench research makes a spectacular showing at the bedside. Findings such as these lead us to wonder if the ardent gaze of academic medicine should be so firmly fixed on large population-based clinical trials. Megatrials are suited to revealing small differences in outcomes in a large number of patients. Yet, in the great wash of generalizable results, rarer but dramatic findings in small subsets of patients may be swept, wastefully, into the oblivion of negative findings.
For researchers and for industry, the search for magic bullets will always hold the allure of altruistic satisfaction, prestige and financial reward. But, as our knowledge of genetic variability in disease processes advances, so too will the potential to develop genetically targeted therapies. As new molecular controls for cell growth, inhibition, apoptosis and other functions are discovered, new compounds to impede or assist these functions will be developed. At the same time, clinical research strategies will change. Each new discovery will require that previous clinical trials be repeated using the new information to select and stratify study subjects. A logical (but not necessarily feasible) consequence of this will be a proliferation of clinical trials. Not only new agents will require evaluation, but previously proven treatments may need to be re-analyzed after patients in the newly identified subgroups are excluded. It will also be important to examine, as did the gefitinib authors, subgroups of patients who showed markedly positive or negative responses to therapy. Biological samples taken during previous RCTs may need to be studied, patients reclassified and analyses repeated.

The new mode of research raises ethical issues. Drugs that are suitable for only small subgroups of patients such as those with specific single somatic mutations - may be of little interest to manufacturers. ${ }^{4}$ Abandonment by industry, if it occurs, will result in more and more "orphan" diseases in need of research funding from public agencies and private foundations.

Molecular medicine will also raise ethical questions for clinicians. In light of the results in 9 patients with previously intractable non-small-cell lung cancer, should all patients with newly diagnosed and advanced non-small-cell lung cancer be offered genetic testing and, potentially, a trial of gefitinib? Patients with the financial resources (the tests and drug are expensive) will likely seek out this new therapy. Should provincial governments fund it? Should physicians recommend it? Such questions, once esoteric, are now becoming urgent. - CMAf

\section{References}

1. Lynch TJ, Bell DW, Sordella R, Gurubhagavatula S, Okimoto RA, Brannigan $\mathrm{BW}$, et al. Activating mutations in the epidermal growth factor receptor underlying responsiveness of non-small-cell lung cancer to gefitinib. $N$ Engl 7 Med 2004;350(21). [Accessed online at www.nejm.org on May 14, 2004]

2. Choi S. Gefitinib. CMA7 2004;170(12):1787.

3. Paez JG, Jänne PA, Lee JC, Tracy S, Greulich H, Gabriel S et al. EGFR mutations in lung cancer: correlation with clinical response to gefitinib therapy. Science Express Reports 29 April 2004.

4. Marx J. Why a new cancer drug works well, in some patients. Science 2004;304(5671):658-9. 